\section{Fluorescent timer}

There are many ways to monitor the onset of gene expression, but so far it has been impossible to detect its downregulation. This problem might have been solved now, as Terskikh and colleagues report in Science a simple method to follow promoter activity.

Last year, a red fluorescent protein (drFP583) was identified in tropical corals, further increasing the wide spectrum of possibilities to light up cells in different colours. Not satisfied with just one colour, Terskikh and colleagues introduced random mutations into drFP583, and found one mutant (called E5) that changes its fluorescence from green to red in a time-dependent manner. As E5 switches from green to red fluorescence over time, it can be used as a timer for gene expression. During the first hours of activity of a promoter, green fluorescence is predominant, whereas sustained activity of the promoter leads to a mixture of green and red fluorescence. A few hours after the promoter is turned off, only red fluorescence remains.

Terskikh and colleagues verified these predictions in three experimental systems. First they monitored up- and down-regulation of E5 expression in Tet-on and Tet-off mammalian expression systems. Then they followed the activity of a heat-shock promoter during heat-induced

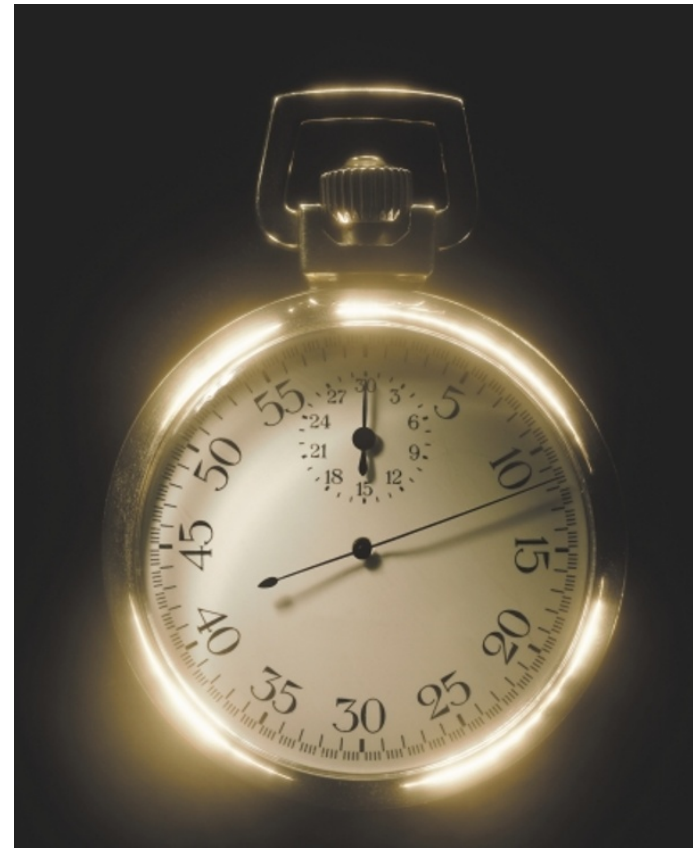

stress of Caenorhabditis elegans. Last, they traced the expression of a homeobox gene involved in the patterning of anterior structures in Xenopus laevis. In all cases, green fluorescence correctly indicated the onset of gene expression and was replaced with red fluorescence when expression ceased. So after decades of blue-stained embryos, we'll now have to get used to seeing gene expression in green and red.

Raluca Gagescu

\section{(D) References and links}

ORIGINAL RESEARCH PAPER Terskikh, A. et al. "Fluorescent timer": protein that changes color with time. Science 290, 1585-1588 (2000)

\section{MEMBRANE DYNAMICS}

\section{Variation on a theme}

When cells are starving, they can eat almost anything, even their own proteins and organelles. This desperate act - called autophagy - involves the engulfment of cytosol and organelles by a membrane that folds back onto itself, giving rise to an autophagosome.

Screens in the yeast Saccharomyces cerevisiae identified many Apg mutants defective in autophagy, and their characterization is progressing rapidly. About two years ago, Ohsumi and colleagues characterized a ubiquitin-like protein modification necessary for autophagy. Two papers from the same group now describe a second, more unusual, ubiquitin-like modification required for this process.

Apg8 is essential for autophagy in yeast, and starvation increases its transcription. Its weak homology with the ubiquitin-like protein
Apg12p suggests that Apg8p might also be a ubiquitin-like protein. Kirisako and colleagues showed that the carboxyl terminus of newly synthesized Apg8p is proteolytically cleaved by the cysteine protease Apg4p. The cleaved form of Apg8p is covalently modified on its terminal glycine and, as a consequence, becomes tightly membrane associated. Ichimura and colleagues determined the

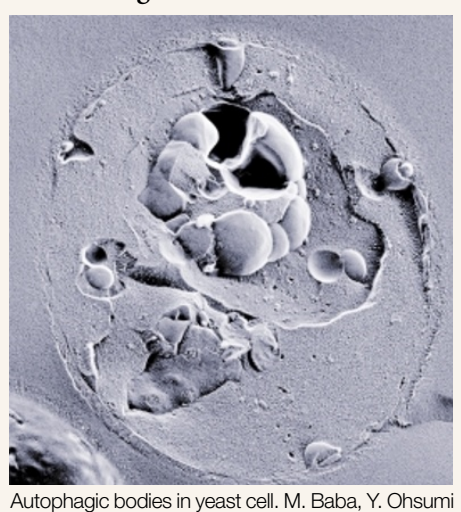

H I G H L I G H T S

\section{WEB WATCH}

Smart by name...

Modular protein domains are nature's solution for building versatile proteins from ready-made building blocks, but keeping track of how they've been shuffled is no easy task. Simple Modular Architecture Research Tool (SMART), written by Peer Bork's group at the

European Molecular Biology Laboratory in Heidelberg, is a powerful tool for putting domains in context.

The simplest way to familiarize yourself with SMART's database of over 400 characterized domains is to click on an entry in the list. This categorizes the occurrence of each domain according to evolutionary distribution and cellular location, as well as including information on structure and function. You can also do sequence alignments.

More specific searches can be done on two levels: sequence or architecture. You can simply paste in a sequence or type in accession numbers from al the commonly used protein databases. If you already know what modular domains your protein of interest contains, you can use the architecture tool to search for proteins that contain the same

combination of domains.

You can also narrow your search down to a particular organism or group of organisms.

SMART can also represent groups of proteins as 'beads on a string' cartoons - an effective way to compare the architecture of a group of proteins. All the 'beads' in these cartoons are clickable, taking you through to that domain's page.

Data entry is a bit clumsy (for instance, the Boolean operators only work if you type them in upper case), but it's worth perservering; even if you don't have a specific query, half an hour spent playing with SMART will reveal nature's uncanny capacity to reuse the same module in many contexts.

Cath Brooksbank 